\title{
Fratura diafisária de tíbia e fíbula em atletas
}

Tibial and fibular diaphyseal fractures in athletes

Fracturas diafisarias tibiales y fibulares en atletas

Paulo Arthur Mendes Milhomem*, Bruno Magalhães Gomes Macedo1, Elisa Guimarães de Figueiredo', Lucas Antonio de Lacerda e Silva', Luisa Lustosa Cabral de Paula Motta', Thaís Cançado Leite', Sérvulo Wallner Pitangui Filho², João Carlos Pereira Salomão ${ }^{3}$.

\section{RESUMO}

Objetivo: Relatar a fratura diafisária de tíbia e fíbula no cenário esportivo, trazendo particularidades do esporte profissional e da escolha do tratamento aplicada ao contexto. Revisão bibliográfica: A fratura de tíbia e fíbula é uma lesão de incidência relevante e requer atenção especial em atletas, realizada por meio da escolha do tratamento e do retorno precoce às atividades. Deve-se atentar ao mecanismo de lesão e classificar adequadamente a fratura para indicar o tratamento. Os exames de imagem são importantes para identificar lesões associadas e evitar armadilhas. O retorno ao esporte é multifatorial e depende da gravidade da lesão e da abordagem utilizada para o tratamento. Entretanto, apenas parte dos atletas alcançam o mesmo nível pré-lesão. Considerações finais: Um diagnóstico preciso com medidas iniciais de tratamento em tempo adequado é crucial. A haste intramedular é uma boa escolha de tratamento e, aliada a reabilitação, apresenta bons resultados. A liberação precoce do apoio e o início imediato da fisioterapia são facilitados por esta modalidade operatória, permitindo que o cuidado do atleta seja otimizado.

Palavras-chave: Fraturas da tíbia, Traumatismos em atletas, Procedimentos ortopédicos, Retorno ao esporte, Fraturas de estresse.

\begin{abstract}
Objective: To report tibial and fibular shaft fractures in the sports scenario, bringing particularities of professional sport and the choice of treatment applied to the context. Literature review: Tibial and fibula shaft fractures have high incidence and require special attention in athletes, done through the right treatment and early return to activities. The lesion mechanism and the proper classification are important to choose the right treatment. Image exams are important to identify other lesions and prevent complications. The return to practice is multifactorial and depends on the severity of the lesion and the treatment approach. However, only part of the athletes reaches the same pre-injury level. Final considerations: An accurate diagnosis with initial treatment measures in a timely manner is crucial. The intramedullary nail is a good treatment choice and, combined with rehabilitation, presents good results. The early support release and the immediate start of physical therapy are facilitated by this operative modality, allowing the athlete's care to be optimized.
\end{abstract}

Keywords: Tibial fractures, Athletic injuries, Orthopedic procedures, Return to sport, Fractures' stress.

\footnotetext{
1 Pontifícia Universidade Católica de Minas Gerais (PUC Minas), Belo Horizonte - MG.

${ }^{*}$ E-mail: pammilhomem@gmail.com

2 Universidade Federal de Minas Gerais (UFMG), Belo Horizonte - MG.

${ }^{3}$ Departamento Médico do América Futebol Clube, Belo Horizonte - MG.
}

SUBMETIDO EM: 7/2020

ACEITO EM: 7/2020

PUBLICADO EM: 10/2020 


\section{RESUMEN}

Objetivo: Informar sobre fracturas de la diáfisis tibial y del peroné en el escenario deportivo, aportando particularidades del deporte profesional y la elección del tratamiento aplicado al contexto. Revisión bibliográfica: La fractura de tibia y peroné es una lesión relevante y requiere atención especial en deportistas, realizada con la elección del tratamiento y el regreso temprano y seguro a las actividades. Se debe prestar atención al mecanismo de lesión y clasificar adecuadamente la fractura para indicar el tratamiento. Los exámenes de imágenes son importantes para identificar lesiones asociadas y evitar dificultades. El regreso al deporte es multifactorial y depende de la gravedad de la lesión y del enfoque utilizado para el tratamiento. Sin embargo, pocos deportistas alcanzan el mismo nivel previo a la lesión. Consideraciones finales: Es crucial un diagnóstico preciso con medidas de tratamiento iniciales de manera oportuna. El clavo intramedular es una buena opción de tratamiento y, combinado con rehabilitación, presenta Buenos resultados. La liberación temprana del soporte y el inicio inmediato de la fisioterapia son facilitados por esta modalidad operativa, lo que permite optimizar la atención del deportista.

Palabras clave: Fracturas de la tibia, Traumatismos em atletas, Procedimientos ortopédicos, Volver al deporte, Fracturas por estrés.

\section{INTRODUÇÃO}

A tíbia é um osso tubular longo que está em disposição vertical na perna e possui formato triangular com três faces e três margens: medial, lateral/interóssea e posterior. A superfície ântero-medial da tíbia e sua crista anterior são facilmente palpáveis, desde a área da tuberosidade anterior até o maléolo medial. $O$ alargamento da fíbula em ambas as extremidades propicia uma maior área de articulação e sustentação do peso. A fíbula situa-se póstero lateralmente à tíbia e está firmemente fixada a ela pela sindesmose tibiofibular, que inclui a membrana interóssea, sua função principal é a fixação muscular, sendo o local de fixação distal (inserção) de um músculo e fixação proximal (origem) de oito músculos (MOORE KL, et al., 2014).

O compartimento anterior da perna contém os músculos tibial anterior, responsável pela flexão dorsal e inversão do pé, extensor longo dos dedos, extensor longo do hálux e o fibular terceiro, responsáveis, primariamente, pela dorsiflexão do pé e tornozelo, além do nervo fibular profundo, artéria e veias tibiais anteriores. Devido a sua localização superficial, pode estar sujeito a síndrome compartimental, tanto nas fraturas expostas como nas fechadas. No compartimento lateral estão localizados os músculos fibulares curto e longo, além do nervo fibular superficial. O compartimento posterior superficial contém a musculatura flexora plantar do pé, formado pelos músculos gastrocnêmio, sóleo e plantar, mas não contém estruturas neuro vasculares importantes. O compartimento posterior profundo contém os músculos tibial posterior, flexor longo do hálux e flexor longo dos dedos, responsáveis pela flexão plantar e inversão, além do nervo tibial e artérias e veias tibiais posteriores e fibulares (NETTER FH, 2012).

A nutrição sanguínea da diáfise tibial provém da artéria nutrícia, ramo da artéria tibial anterior ou posterior e dos inúmeros vasos periosteais. A artéria nutrícia perfura o músculo tibial posterior, para o qual envia ramos, e entra no forame nutrício no terço proximal da face posterior da tíbia. O nervo fibular comum que segue na tela subcutânea através da face lateral da cabeça e colo da fíbula é o nervo subcutâneo mais frequentemente lesado devido ao seu trajeto superficial (MOORE KL, et al., 2014).

A fratura diafisária da tíbia é a mais comum dos ossos longos estando sujeita a várias complicações. Estima-se que a incidência desse tipo de lesão em 16.9/100.000 pessoas/ano, acometendo principalmente o sexo masculino, entre 10 e 20 anos, com incidência de 21.5/100.000 pessoas/ano. No sexo feminino, o perfil de indivíduos acometidos é, principalmente, de mulheres entre 30 a 40 anos com incidência de 12.3/100.000 pessoas/ano (LARSEN P, et al., 2015). Dentre as lesões do esporte, esse tipo de fratura merece atenção pois, além da alta incidência ( $4 \%$ de todas as fraturas ligadas ao esporte), demanda grande tempo de afastamento das atividades devido a possibilidade de complicações, bem como lesões associadas e sequelas (ROBERTSON GAJ, 2016). 
As fraturas dos ossos da perna podem evoluir com lesões graves e incapacitantes, podendo ser causadas por mecanismos de baixa e alta energia, com distintos níveis de gravidade. Ainda, essa é uma região onde comumente se diagnostica fratura por estresse, outro mecanismo englobado no complexo destas fraturas que é muito comum em atletas (ASTUR DC, et al., 2016). No meio esportivo, as fraturas fechadas são mais prevalentes, porém as lesões expostas podem estar presentes e possuem prognóstico mais complicado.

A classificação da lesão é importante para determinar a gravidade e indicar a forma de tratamento adequado. Devemos utilizá-las de forma específicas de acordo com as características da lesão, de maneira a indicar para o ortopedista os perigos potenciais e ajudar na determinação do tratamento mais apropriado. Deve-se atentar sempre ao mecanismo de lesão da fratura e, por meio de avaliação clínica e radiológica criteriosa, avaliar a possibilidade de ocorrência de outras lesões concomitantes. A presença do médico no momento do trauma pode contribuir para um atendimento mais seguro e eficaz. A escolha entre o tratamento, cirúrgico ou conservador, vai depender da gravidade e do tipo de fratura. A intervenção cirúrgica comumente é o tratamento de escolha em atletas pois, no esporte, é importante considerar também o tempo de afastamento das atividades, o qual pode influenciar na perda de condicionamento cardiorrespiratório, muscular e propriocepção. Além disso, o tratamento conservador tem uma maior taxa de pseudoartrose quando comparado ao procedimento cirúrgico (BOULTON C e O'TOOLE RV, 2017).

O objetivo deste presente estudo é abordar a fratura de ossos da perna dentro do cenário do esporte, priorizando o atendimento e a reabilitação precoces de acordo com as necessidades dos atletas. É importante citar que a condução de um paciente não esportista pode diferir da abordagem de um atleta profissional, já que este possui maior cobrança relacionada a sua recuperação e eficiência, além de infraestrutura de qualidade e melhor assistência, que sustentam o seu tratamento.

\section{REVISÃO BIBLIOGRÁFICA}

A fratura de diáfise de tíbia e fíbula possui maior incidência em jovens do sexo masculino com idade entre 15 e 19 anos, sendo esta de 109/100.000 pessoas/ano (EGOL KA, et al., 2020). Dentre os mecanismos de lesão, os traumas de alta energia possuem maior prevalência, com destaque para o acidente automobilístico. Observa-se uma redução na incidência de politraumatismo associado, devido a mudanças no padrão de lesão. Já dentre os mecanismos de baixa energia destacam-se a queda da própria altura e as lesões esportivas. O futebol americano apresenta grande incidência deste tipo de lesão, correspondendo a cerca de $80 \%$ das fraturas nesse esporte, segundo estudos ingleses (BOULTON C e O'TOOLE RV, 2017).

A presença do médico no local do trauma é de suma importância para a observação e descrição do mecanismo de lesão, possibilitando assim uma abordagem direta, otimizada e com as melhores condutas possíveis. Esse cenário é comum na rotina de treinos e jogos de grandes clubes esportivos, onde a equipe do departamento médico acompanha atentamente os atletas e oferece o suporte necessário em casos de intercorrências.

Segundo Court-Brown CM (2017), são esportes com alta incidência de lesões, em ordem decrescente: futebol americano, rúgbi, ciclismo de pista/montanhismo e esportes de inverno. No que tange às fraturas da diáfise da tíbia, o padrão de mecanismo de lesão está alterando. Atualmente, $44,9 \%$ das fraturas dessa região são devido à queda da própria altura, e lesões esportivas ainda são comuns. De acordo com Boulton C. e O'toole RV (2017), em 1988-1990, acidentes automobilísticos (37,5\%) tinham mais prevalência nesse tipo de lesão, seguido por lesões esportivas $(30,9 \%)$ e queda da própria altura $(17,5 \%)$. Em relação às fraturas expostas da tíbia, a prevalência está diminuindo, apesar de terem ocorrido em $20,2 \%$ dos casos do estudo. As fraturas isoladas da fíbula são pouco relevantes com tratamento e prognóstico mais simples (COURTBROWN CM, 2017).

Não menos importante, a fratura por estresse, lesão de alta incidência em atletas, ocorre por sobrecargas cíclicas, repetitivas na região, sem um tempo de recuperação suficiente. Esse mecanismo causa microfraturas nas trabéculas ósseas normais, ultrapassando a capacidade de reparo e regeneração. Na tíbia é mais comum em corredores ou em praticantes de esportes com saltos. Além disso, essas lesões são classificadas em baixo ou alto risco. As de baixo risco acometem a cortical póstero medial da tíbia e são as mais comuns. 
Estas, apresentam melhor prognóstico e geralmente o tratamento conservador é o de escolha. Em relação às de alto risco, as quais acometem a cortical anterior da tíbia, são mais raras, têm pior prognóstico e muitas vezes há indicação de tratamento cirúrgico (ASTUR DC, et al., 2016; MILLER TL e KOEDING CC, 2017).

A classificações têm como objetivo descrever as características das lesões, avaliando fatores como o acometimento de tecidos moles, localização anatômica da fratura, quantidade e localização de fragmentos, assim como angulação e o deslocamento ósseo. Existem diversos tipos de classificações. O sistema mais amplamente utilizado em fraturas da tíbia e fíbula é o AO/OTA (AO Foundation/Orthopaedic Trauma Association). Este sistema diferencia as lesões em 3 tipos: fraturas simples (tipo A), fraturas em cunha (tipo B) e fraturas complexas (tipo C).

Esses tipos se subdividem em 3 grupos que indicam maior gravidade de acordo com sua numeração crescente (de 1 a 3 ). Estes 3 grupos ainda são divididos em três subgrupos dependendo da localização da fratura, nos tipos A e B, podendo ser com a fíbula intacta, tibial e fibular em níveis diferentes ou tibial e fibular no mesmo nível. Para o tipo $\mathrm{C}$, os subgrupos avaliam a quantidade de fragmentos fraturados (MEINBERG $E G$, et al., 2018). A classificação AO mais comum para esse tipo de fratura é a 42-A1 que se caracteriza como uma fratura de tíbia e fíbula, diafisária, simples e em espiral (LARSEN P, et al., 2015).

Existe ainda a classificação de Tscherne que é utilizada para avaliar os danos de partes moles em fraturas fechadas. Esta leva em consideração os mecanismos de lesão indiretos e diretos e subdivide-as em 4 graduações (de 0 a 3) na qual evoluem de um dano mínimo a extenso de tecidos moles adjacentes. Ela deve ser utilizada junto à do sistema AO/OTA para uma abordagem completa (MEINBERG EG, et al., 2018).

A classificação de Gustilo e Anderson é a principal utilizada em casos de fraturas abertas, pós desbridamento, de modo a avaliar a gravidade e o grau de contaminação, principalmente para a tomada de decisão quando a antibioticoprofilaxia. Sua classificação é dividida em 3 graus, sendo o terceiro subdividido em 3 grupos de acordo com a gravidade da lesão (MEINBERG EG, et al., 2018).

Segundo Fields KB (2019), são sinais e sintomas da fratura diafisária da tíbia e fíbula edema localizado, dor e incapacidade de suportar peso. Ainda, em casos de fraturas de grande deslocamento, comumente observadas no esporte, principalmente as causadas por trauma direto, há uma mudança no alinhamento da perna, sendo importante um exame cuidadoso da pele subjacente e de estruturas neurovasculares, especialmente em fraturas expostas. Dessa forma, é importante examinar os pulsos distais e a função motora e sensorial das extremidades inferiores.

O exame de imagem radiográfico é o mais utilizado devido ao seu baixo custo, fácil acesso e tempo de realização pequeno. As radiografias em ântero-posterior (AP) e perfil são determinantes para o diagnóstico desse tipo de fratura e devem ser solicitadas logo que o atleta chega ao hospital de referência. Além disso, devem ser obtidas imagens do joelho e tornozelo para avaliação de possíveis lesões associadas. A tomografia computadorizada (TC) e a ressonância magnética (RM) podem ser realizadas em casos de má visualização da fratura ou suspeita de outras lesões relacionadas.

No caso de suspeita de fratura por estresse, a RM é o exame de imagem com maior sensibilidade e especificidade para o diagnóstico, sendo o exame de escolha. Os exames de imagem são essenciais para uma melhor avaliação do caso e servem como orientação e meio de comparação para o ortopedista desde o primeiro contato com o atleta até a consolidação da fratura e o retorno às atividades (FELDMAN et al., 2016; BOULTON C e O'TOOLE RV, 2017).

O tratamento conservador para fratura de tíbia e fíbula é baseado na redução incruenta e imobilização gessada longa, com ou sem conversão eventual para órteses pré-fabricadas. Na utilização do aparelho gessado ou da órtese, deve-se manter um alinhamento efetivo da fratura, observando os desvios máximos aceitáveis, menores que $10^{\circ}$ em rotação e $10^{\circ}$ em angulação, assim como menos de $5 \mathrm{~mm}$ de gap ósseo (FIELDS KB, 2019). De acordo com Boulton C e O'toole RV (2017), o tratamento conservador é indicado para pacientes de alto risco anestésico, fraturas com alinhamento inicial excelente e que necessitam de pouca ou nenhuma redução para obter um alinhamento apropriado. Esse método tem caído em desuso devido ao avanço das técnicas cirúrgicas. 
O paciente atleta que realiza o tratamento conservador deve ser avaliado semanalmente para a troca do gesso, uma vez que o edema diminui. Ainda, o gesso é mais indicado até a terceira semana devendo ser trocado para uma imobilização mais leve, que permita a deambulação. Na maioria dos casos, utiliza-se um material de fibra de vidro para permitir ao paciente a deambulação com apoio parcial e, posteriormente, apoio total.

O acompanhamento deve ser feito com a utilização de radiografias em série semanalmente, assim é possível identificar qualquer modificação na lesão como a perda da redução ou mudança de posição. A partir da quarta semana, o atleta deve realizar acompanhamento quinzenal até que haja evidência radiológica de consolidação, como a presença de calo ósseo e preenchimento da linha de fratura.

Após a cura, novos exames radiográficos só devem ser realizados em caso de dores incomuns no local ou novo trauma. Por fim, o atleta deve estar ciente que o processo de cicatrização óssea requer um tempo maior e que pode haver alguns sintomas persistentes (FIELDS KB, 2019).

A intervenção cirúrgica é o tratamento de escolha para a maioria dos casos de fratura desviada de tíbia e fíbula, já que o tratamento conservador está associado a piores resultados com referência à ocorrência de pseudoartrose e consolidação viciosa. O uso de haste intramedular bloqueada (HIM) é a técnica de tratamento mais comumente utilizada para esse tipo de lesão em atletas, sendo indicada tanto para fraturas fechadas quanto para expostas de baixo grau. Uma das principais vantagens deste método é a liberação do apoio precoce, possibilitando acelerar o processo de reabilitação. Essas questões são extremamente importantes no cenário esportivo e influenciam na escolha do tratamento.

Outra opção de abordagem cirúrgica para o tratamento de fraturas diafisárias da tíbia no esporte é a osteossíntese com placa, técnica comumente utilizada para fraturas proximais e distais. Nestes tipos de fratura, torna-se difícil a introdução de hastes e garantir uma estabilidade mecânica. Para fraturas do terço médio da tíbia, o uso de placas fica reduzido para os casos nos quais a utilização da haste seja impossibilitada ou diminuída.

São exemplos dessa situação casos em que a tíbia é muito estreita para a haste ou quando o canal medular está obstruído, seja por calo ósseo ou em pacientes cuja introdução anterógrada da haste esteja impossibilitada, como em portadores próteses totais do joelho (BOULTON C e O'TOOLE RV, 2017).

No período pós-operatório, deve-se atentar a potenciais complicações e medidas para preveni-las. Dor anterior no joelho, mau alinhamento da fratura e consolidação viciosa podem ser evitadas com tratamento e acompanhamento adequados. É importante estar atento quanto à possibilidade de infecção do sítio cirúrgico e à síndrome compartimental aguda (SCA) que é caracterizada por sinais e sintomas que podem incluir dor persistente e progressivo edema tenso, além de dor com flexão e extensão passivas dos dedos dos pés (FIELDS KB, 2019).

A SCA possui uma taxa de ocorrência relativamente alta, variando entre 1,5 - 11\% e que, quando não diagnosticada, poderá resultar em consequências clínicas graves além de elevar os custos associados ao tratamento da fratura (BOULTON C e O'TOOLE RV, 2017).

Dentre os pacientes abordados cirurgicamente, Robertson GAJ, et al. (2016) demonstraram que em média de $33 \%$ necessitam de reintervenções, sendo maior na técnica de HIM (34\%). Entre as complicações mais comuns, 33\% apresentam síndrome compartimental, 10\% infecção do sítio operatório e $20-54 \%$ apresentam dor no joelho pós-operatória. Já nas abordagens não cirúrgicas, as taxas de reintervenção são em média de $19 \%$ dos casos.

No período pós-operatório inicial, os atletas devem ser avaliados semanalmente, para prevenção ou diagnóstico precoce de possíveis complicações, com realização de radiografias seriadas para 0 acompanhamento da consolidação e certificação da ausência de perda da redução óssea. Podemos esperar uma consolidação satisfatória da fratura em cerca de seis semanas, e em pacientes diabéticos ou tabagistas esse tempo pode ser prolongado. 
Em atletas, o acompanhamento com fisioterapeuta especializado é essencial e deve ter início imediato, com medidas anti-inflamatórias iniciais, prevenção de rigidez articular e ganho de amplitude de movimento, assim como exercícios isométricos. Nos casos submetidos a tratamento cirúrgico com HIM temos como vantagem a liberação do apoio precoce, permitindo acelerar a recuperação com uma progressão mais rápida no processo de reabilitação. Após a consolidação óssea, a progressão para exercícios com carga de forma excêntrica e concêntrica está liberada. Na última fase antes do retorno completo às atividades, é realizado o treino funcional, com a prática do gestual esportivo específico.

O retorno ao esporte é multifatorial e depende da gravidade da lesão e da abordagem utilizada para o tratamento. Dessa maneira, a classificação de Tscherne e o AO/OTA podem ser utilizados como preditores desse retorno. De acordo com Robertson GAJ, et al. (2016), a retomada das atividades físicas em pacientes cirúrgicos foi de $91,5 \%$, já entre os pacientes que se submeteram a abordagens mais conservadoras as taxas de retorno foram de $66,7 \%$.

Ainda, a escolha do tipo de tratamento reflete no tempo de retorno ao esporte, sendo ele maior em abordagens mais conservadoras (> 100 semanas). Dentre as técnicas cirúrgicas, as que apresentaram maiores taxas de retorno foram a redução aberta e fixação interna (ORIF) e o uso de haste intramedular bloqueada (HIM), no entanto a primeira apresenta maior tempo para o retorno quando comparada à segunda - 52 semanas para 41 semanas.

Além disso, o retorno está relacionado com a gravidade da lesão, logo, atletas que apresentam lesões abertas e com a classificação Tscherne elevadas irão apresentar maior tempo de recuperação e menores taxas de retorno. Ainda, segundo Fields KB (2019), o retorno para a atividade esportiva total depende das características do paciente, como idade, comorbidades prévias, gravidade da fratura da tíbia, presença de lesões concomitantes e nível de atividade pré-lesão.

De forma geral, para pacientes que possuem ocupações que exigem pouco esforço de caminhada ou de apoio, o retorno às atividades se dá à medida que o tratamento com gesso cruropodálico progride. Já para atletas, o retorno ao treinamento sem carga de peso acontece em média de 4 a 6 semanas. Em adição, é válido ressaltar que apesar da maioria dos atletas retornar ao esporte, apenas uma parte destes irá apresentar o mesmo nível de desempenho que apresentava antes da lesão.

\section{CONSIDERAÇÕES FINAIS}

A fratura diafisária de tíbia e fíbula é uma lesão de incidência relevante na população e requer atenção especial nos atletas, entre os quais a escolha do método de tratamento leva em consideração o retorno precoce e seguro às atividades. O estudo em questão contribui para a otimização da abordagem clínica e cirúrgica frente à lesão, norteando, assim, a conduta médica diante dos atletas, com a intenção de atualizar o ortopedista e proporcionar ao paciente um tratamento baseado nas mais recentes evidências. Devemos salientar que o tema em discussão apresenta escasso volume literário devido, sobretudo, à sua especificidade de abordar pacientes atletas, sendo necessárias adaptações para o cenário esportivo. É importante enfatizar a necessidade de um diagnóstico preciso, assim como as medidas iniciais para evitar danos secundários ou piora do quadro, além do tratamento no tempo adequado. A intervenção cirúrgica por meio de HIM tem se mostrado a melhor opção de tratamento e, aliada a uma reabilitação adequada, tende a apresentar resultados positivos e satisfatórios. Vale ressaltar que a liberação precoce do apoio e o início imediato da fisioterapia são ferramentas facilitadas por esta modalidade cirúrgica, permitindo que o cuidado do atleta seja otimizado.

\section{REFERÊNCIAS}

1. ASTUR DC, et al. Fraturas por estresse: definição, diagnóstico e tratamento. Revista Brasileira de Ortopedia, 2016; 51(1): 3-10.

2. BODEN BP, et al. Tibia and fibula fractures in soccer players. Sports medicine, 1999; 7: 262-266. 
3. BOULTON C, O'TOOLE RV. Fraturas das diáfises da tíbia e fíbula. In: ROCKWOOD; GREEN. Fraturas em Adultos. 8. ed. Barueri: Manole, 2017; 57: 2361-2419.

4. CHANG WR, et al. Tibial shaft fractures in football players. Journal of Orthopaedic Surgery and Research, $2007 ; 2: 11$.

5. COURT-BROWN CM. Epidemiologia das fraturas e luxações. In: ROCKWOOD; GREEN. Fraturas em Adultos. 8. ed. Barueri: Manole, 2017; 3: 58-106.

6. COURT-BROWN CM, McBIRNIE J. The epidemiology of tibial fractures. The Journal of Bone and Joint Surgery, 1995; 77B (3): 417-42.

7. DEN OUTER AJ, et al. Conservative versus operative treatment of displaced non comminuted tibial shaft fractures. A retrospective comparative study. Clin Orthop Relat Res, 1990; 252: 231-237.

8. EGOL KA, et al. Handbook of Fractures. 6. ed. New York: Wolters Kluwer, 2020; 37: 458-467.

9. FELDMAN JJ, et al. Tibial Stress Fractures in Athletes. Orthop Clin Am, 2016; 47: 733-741.

10. FERREIRA JCA, et al. Estudo comparativo entre o aparelho e haste intramedular bloqueada no tratamento da fratura fechada da diáfise da tíbia. Revista Brasileira de Ortopedia, 2006; 41(10): 405-10.

11. FERREIRA JCA. Fraturas da diáfise dos ossos da perna. Revista Brasileira de Ortopedia, 2000; 35(10): $375-383$.

12. FIELDS KB, et al. Tibial shaft fractures in adults - 2019. Post TW, ed. UpToDate. Waltham, MA: UpToDate Inc.

13. FRANCISCO AC, et al. Comparison of Soccer Shin Guards in Preventing Tibia Fracture. Am J Sports Med, 2000; 28(2): 227-33.

14. KOJIMA KE, FERREIRA RV. Fraturas da diáfise da tíbia. Revista Brasileira de Ortopedia, 2011; 46(2): 130-5.

15. LABRONICI PJ, et al. Fatores que afetam a consolidação óssea após tratamento com haste intramedular bloqueada e placa em ponte nas fraturas diafisárias da tíbia. Revista Brasileira de Ortopedia, 2007; 42(5): 139-145.

16. LARSEN P, et al. Incidence and epidemiology of tibial shaft fractures. International Journal of the Care of the Injured, 2015; 46(4): 746-750.

17. MEINBERG EG, et al. Fracture and Dislocation Classification Compendium. Journal of Orthopaedic Trauma, 2018; 32: S1-S10.

18. MILLER TL, KOEDING CC. Fraturas por estresse. In: ROCKWOOD; GREEN. Fraturas em Adultos. 8. ed. Barueri: Manole, 2017; 21: 630-644.

19. MOORE KL, et al. Anatomia Humana Orientada para a Clínica. 7 ed. Rio de Janeiro: Koogan, 2014.

20. NELSON GE, et al. Blood supply of the human tibia. J Bone Joint Surg [AM], 1960; 42: 625-635.

21. ROBERTSON GAJ, WOOD AM. Return to Sport After Tibial Shaft Fractures: A Systematic Review. Sports Health, Edinburgh, 2016; 224-230.

22. SHINDLE MK, et al. Stress Fractures About the Tibia, Foot, and Ankle. J Am Acad Orthop Surg, 2012; 20(3):167-76.

23. THOMPSON JC. Netter, Atlas de Anatomia ortopédica. 2. ed. Rio de Janeiro: Elsevier Editora Ltda, 2012. 